TMUP-HEL-9702

hep-ph/9701362

\title{
Can Long-Baseline Neutrino Oscillation Experiments Tell Us about Neutrino Dark Matter?
}

\author{
Hisakazu Minakata and Yoriaki Shimada \\ Department of Physics, Tokyo Metropolitan University \\ Minami-Osawa, Hachioji, Tokyo 192-03, Japan
}

(January 1997)

\begin{abstract}
We define the ratio $R=$ (No. of observed electron events)/ (No. of expected muon events - No. of observed muon events) as a measure for relative importance of $\nu_{\mu} \rightarrow \nu_{e}$ and $\nu_{\mu} \rightarrow \nu_{\tau}$ oscillations which will be measurable in coming long-baseline neutrino experiments. We then argue that if neutrino oscillation is observed with $0.02<R<0.87$ it implies the rejection of the neutrino dark matter hypothesis. Our argument is valid if neutrinos come with only three flavors without steriles and if they have a hierarchical mass pattern.

14.60.Pq
\end{abstract}

Typeset using REVTEX 
The possibility of neutrino dark matter is actively investigated both from theoretical and experimental points of view. The cold and hot dark matter cosmology is one of the rival models which can account for structure formation in the universe [1]. The hot dark matter is the indispensable ingredient in the scenario by which the normalization of the density fluctuation required by structure formation can be made consistent with the COBE observation [2]. The neutrinos with the mass range of $2-20 \mathrm{eV}$ are the best known candidate for the hot dark matter.

The neutrinos with dark matter mass scale are subject to the active experimental searches. The ongoing short-baseline experiments, CHORUS [3] and NOMAD [4], look for evidence for the neutrino oscillation $\nu_{\mu} \rightarrow \nu_{\tau}$ with the sensitivity $\sin ^{2} 2 \theta \simeq 3 \times 10^{-4}\left(3 \times 10^{-3}\right)$ for the mass range $m_{\nu_{\tau}} \gtrsim 100(10) \mathrm{eV}$. Moreover, the new experiment COSMOS with greater sensitivity are planned and may be able to run at sometime early in the next century.

In this paper we address a question which is rarely asked; can we learn about dark matter neutrinos from the long-baseline neutrino oscillation experiments? An immediate answer to this question is likely to be "clearly no". The principal objective of the longbaseline neutrino experiments is to confirm the large-angle mixing of either $\nu_{\mu} \rightarrow \nu_{\tau}$ or $\nu_{\mu} \rightarrow \nu_{e}$ which is strongly suggested by the atmospheric neutrino anomaly [0]7]. Hence they are designed to probe the mass-squared difference of the order of $\Delta m^{2} \simeq 10^{-2} \mathrm{eV}^{2}$. These mass difference seems to be too tiny to probe the dark matter mass scale $\sim 10 \mathrm{eV}$, apart from the effects of large $\Delta m^{2}$ averaged over many period.

Nevertheless, we are going to claim in this paper that the long-baseline neutrino oscillation experiments do probe the dark matter mass neutrinos.

Our original motivation for this investigation started with the following question. Suppose that CHORUS and NOMAD or even future new experiments fail to observe the appearance events $\nu_{\mu} \rightarrow \nu_{\tau}$. Can we conclude from these results that the hypothesis of $\tau$-neutrino dark matter is excluded? The answer to this question is no for one obvious and one notso-obvious reasons. The obvious one is that it can exist with smaller mixing angle which is outside of the sensitivities of these experiments. The non-obvious reason, which is more 
serious, is that in the three-flavor mixing framework there is a region of mixing parameters, a large- $s_{13}^{2}$ region, that cannot be probed by the particular appearance experiment $\nu_{\mu} \rightarrow \nu_{\tau}$ but is allowed by all the accelerator and the reactor experiments. This fact is noticed and displayed in Fig. 12 of [10]. The latter reasoning is more serious because we cannot conclude for sure that the dark matter $\tau$ neutrino does not exist no matter how the sensitivity of the experiment is improved.

These considerations naturally lead us to the question if additional constraints on dark matter neutrinos could emerge from other experiments in an unexpected way. In this context, we examine the long-baseline neutrino oscillation experiments which might be able to tell something about the dark matter neutrinos. We show in this paper that if the neutrinos come only with three flavors, i.e., without steriles, the long-baseline experiments can exclude the dark matter neutrino hypothesis unless their masses are strongly degenerate. We emphasize that to make such statement meaningful it is essential for the long-baseline experiments to have capability of doing detection not only in the $\nu_{\mu} \rightarrow \nu_{e}$ appearance channel but also in the disappearance channel.

Since our conclusion heavily depends upon the theoretical framework let us define precisely what is the mass pattern and the three-flavor mixing framework with which we work throughout this paper. We assume that there exist three flavor of neutrino states with the mass hierarchy either $m_{3} \gg m_{1} \approx m_{2}$ or $m_{1} \approx m_{2} \gg m_{3}$. In each case $\left|\Delta M^{2}\right| \equiv\left|\Delta m_{13}^{2}\right| \approx\left|\Delta m_{23}^{2}\right|$ and is of the order of $5-100 \mathrm{eV}^{2}$. There is only one remaining mass difference, $\Delta m^{2} \equiv \Delta m_{12}^{2}$, which can be used as the solar or as the atmospheric neutrino mass scale. If we choose the former option we will not be able to detect any signals in the long-baseline experiments and hence no useful constraint will be obtained. For this reason we employ the alternative option $\Delta m^{2} \simeq 10^{-2} \mathrm{eV}^{2}$, and assume that the experiments do observe the neutrino oscillations. Thus, we cannot accommodate the solar neutrino solution to our restrictive framework of three-flavor mixing enriched by mass scales suggested by the dark matter and the atmospheric neutrinos. We will return this point at the end of this paper. 
For definiteness we use the standard form of the Cabibbo-Kobayashi-Maskawa mixing matrix

$$
U=\left[\begin{array}{ccc}
c_{12} c_{13} & s_{12} c_{13} & s_{13} e^{-i \delta} \\
-s_{12} c_{23}-c_{12} s_{23} s_{13} e^{i \delta} & c_{12} c_{23}-s_{12} s_{23} s_{13} e^{i \delta} & s_{23} c_{13} \\
s_{12} s_{23}-c_{12} c_{23} s_{13} e^{i \delta} & -c_{12} s_{23}-s_{12} c_{23} s_{13} e^{i \delta} & c_{23} c_{13}
\end{array}\right]
$$

for the neutrino mixing matrix $\nu_{\alpha}=U_{\alpha j} \nu_{j}(\alpha=e, \mu, \tau$ and $j=1,2,3)$.

If one of the two independent $\Delta m^{2}$ is of the order of dark matter mass scale the mixing angles are subject to the stringent constraint by the data of accelerator and reactor experiments. The clear recognition of this point in a general three-flavor setting has started with the works in refs. [8,9], and the quantitatively accurate bound is worked out by Fogli, Lisi, and Scioscia [10] in the explicit CKM parametrization as ours (11). The resultant allowed parameter region can be plotted on $s_{13}^{2}-s_{23}^{2}$ plane. (See Fig. 2.) There exist three allowed regions

(i) small- $s_{13}^{2}$ and small- $s_{23}^{2}$

(ii) large- $s_{13}^{2}$ and arbitrary $s_{23}^{2}$

(iii) small- $s_{13}^{2}$ and large- $s_{23}^{2}$

It is of key importance to recognize that the tightly constrained region by short-baseline experiments can naturally lead to sizable oscillations at long-baseline [8]. Moreover, each allowed region (i)-(iii) corresponds to the pure two-flavor oscillation channels. Namely, the parameter regions (i), (ii) and (iii) correspond to almost pure $\nu_{\mu} \rightarrow \nu_{e}, \nu_{\mu} \rightarrow \nu_{\tau}$, and $\nu_{e} \rightarrow \nu_{\tau}$ oscillation channels, respectively.

Then, it is very simple (but is crucial) to observe that any indication of genuine threeflavor mixing signals the failure of the dark matter mass hypothesis defined above. This is the task that the long-baseline neutrino oscillation experiments can do.

To quantify the point let us focus on the particular type of the long-baseline experiment that can measure the appearance channel $\nu_{\mu} \rightarrow \nu_{e}$ as well as the disappearance channel, i.e., 
$\nu_{\mu}$ beam attenuation. We note that the experiment KEK-PS $\rightarrow$ Super-Kamiokande, the only experiment that is already funded and will run in early 1999, is of this type.

How can one characterize quantitatively the genuine three-flavor nature of the oscillation? We make a concrete proposal here. We propose the quantity

$$
\begin{aligned}
R & =\frac{\text { No. of observed electron events }}{\text { No. of expected muon events }- \text { No. of observed muon events }} \\
& =\frac{\int d E_{\nu} f\left(E_{\nu}\right) \sigma_{c c}^{\left(\nu_{e}\right)}\left(E_{\nu}\right) P\left(\nu_{\mu} \rightarrow \nu_{e}\right)}{\int d E_{\nu} f\left(E_{\nu}\right) \sigma_{c c}^{\left(\nu_{\mu}\right)}\left(E_{\nu}\right)\left[1-P\left(\nu_{\mu} \rightarrow \nu_{\mu}\right)\right]}
\end{aligned}
$$

as the appropriate measure for the genuine three-flavor character of the oscillation. In (2) $f\left(E_{\nu}\right)$ indicates the muon neutrino flux at neutrino energy $E_{\nu}$ at the detector, $\sigma_{c c}^{\left(\nu_{x}\right)}$ denotes the charged current cross section of $\nu_{x}$ on targets in water, and $P\left(\nu_{\alpha} \rightarrow \nu_{\beta}\right)$ is the oscillation probability of $\nu_{\alpha} \rightarrow \nu_{\beta}$

If the oscillation is in almost pure $\nu_{\mu} \rightarrow \nu_{e}$ channel the ratio $R$ is close to unity. Whereas if it is in the almost pure $\nu_{\mu} \rightarrow \nu_{\tau}$ channel the ratio $R$ is approximately zero. Since the accelerator and the reactor bounds are so restrictive that tightly constrain the mixing parameters into the pure two-flavor oscillation channel the experimental observation of $R$ not too close to either zero or unity implies the rejection of the dark matter hypothesis. Our remaining task is to quantify this statement, which will be the topics of our subsequent discussions.

We use approximate expressions of neutrino oscillation probabilities in vacuum as

$$
\begin{aligned}
P\left(\nu_{\mu} \rightarrow \nu_{e}\right) & =\frac{1}{2} s_{23}^{2} \sin ^{2} 2 \theta_{13} \\
& +\sin 2 \theta_{12} c_{13}^{2}\left\{\sin 2 \theta_{12}\left(c_{23}^{2}-s_{23}^{2} s_{13}^{2}\right)+\cos 2 \theta_{12} \sin 2 \theta_{23} s_{13} \cos \delta\right\} \sin ^{2}\left(\frac{\Delta m^{2} L}{4 E}\right) \\
& -2 J \sin ^{1}\left(\frac{\Delta m^{2} L}{2 E}\right) \\
1-P\left(\nu_{\mu} \rightarrow \nu_{\mu}\right) & =2 s_{23}^{2} c_{13}^{2}\left(1-s_{23}^{2} c_{13}^{2}\right) \\
& +4\left(s_{12}^{2} c_{23}^{2}+c_{12}^{2} s_{23}^{2} s_{13}^{2}+\frac{1}{2} \sin 2 \theta_{12} \sin 2 \theta_{23} s_{13} \cos \delta\right) \\
& \times\left(c_{12}^{2} c_{23}^{2}+s_{12}^{2} s_{23}^{2} s_{13}^{2}-\frac{1}{2} \sin 2 \theta_{12} \sin 2 \theta_{23} s_{13} \cos \delta\right) \sin ^{2}\left(\frac{\Delta m^{2} L}{4 E}\right)
\end{aligned}
$$


where $\Delta m^{2} \equiv \Delta m_{12}^{2}$ and $J=c_{12} s_{12} c_{23} s_{23} c_{13}^{2} s_{13} \sin \delta$ is the leptonic analogue of the Jarlskog parameter [11]. These formulae are obtained by taking average over the rapid oscillations due to the large arguments in the trigonometric functions, $\frac{\Delta M^{2} L}{4 E}=$ $127\left(\frac{\Delta M^{2}}{1 e V^{2}}\right)\left(\frac{L}{100 k m}\right)\left(\frac{E}{1 G e V}\right)^{-1}$, where $\Delta M^{2} \equiv \Delta m_{13}^{2} \simeq \Delta m_{23}^{2}$.

We focus on the particular experiment KEK $\rightarrow$ Super-Kamiokande and use the muon neutrino flux calculated by Nishikawa et al. [12]. For the cross sections of the chargedcurrent weak interaction we use the value obtained by Nakahata et al. [13]. We assume that the detection efficiency of electrons and muons in Super-Kamiokande is $100 \%$. This is a reasonable assumption because of high lepton energies $\gtrsim 200 \mathrm{MeV}$. For consistency check we have computed the number of events without neutrino oscillation. The expected number of muon events is 460-470 (whose uncertainty reflects errors in reading the neutrino flux off the figure in [12]) for the neutrino flux from $10^{20}$ protons, which roughly corresponds to 12 month's run of the experiment, in rough agreement with the number $\sim 500$ quoted by Nishikawa et al [12].

We here make a comment on the earth matter effect. It is, of course, necessary to take into account the matter effect because it affects the oscillation probabilities at the level of a few to several \% depending upon the mixing parameters. However, we argue that for our purpose vacuum oscillation formula gives a fairly good approximation. The region of parameters which requires great accuracy is the regions with $R \simeq 0$ and $R \simeq 1$. In the former region the $\nu_{\mu} \rightarrow \nu_{\tau}$ oscillation is dominant and it is known that the matter effect is negligibly small in this region. In the latter region with $R \simeq 1$, on the other hand, the ratio $R$ is also insensitive to the matter effect. This is because it affects almost equally to the numerator and the denominator, since they are both dominated by the $\nu_{\mu} \rightarrow \nu_{e}$ oscillation. Therefore, ignoring the matter effect must give a fairly good approximation in computing $R$ in the relevant regions of mixing parameters for our purpose.

In Fig. 1 we present bird's-eye view of the global features of the ratio $R$ and its projection onto the plane spanned by $\tan ^{2} \theta_{13}$ and $\tan ^{2} \theta_{23}$. The remaining angles are fixed as $\theta_{12}=\pi / 4$ 
and $\delta=0 . \Delta m^{2}$ is taken as $10^{-2} \mathrm{eV}^{2}$. The correspondence between various symbols for lines and the equi- $R$ contours are exhibited in the figure. At small values of $\tan ^{2} \theta_{13}$ and $\tan ^{2} \theta_{23}$ $R \simeq 1$ as expected for $\nu_{\mu} \rightarrow \nu_{e}$ dominant region. (Unity of the cross section ratio $\sigma_{c c}^{\left(\nu_{e}\right)} / \sigma_{c c}^{\left(\nu_{\mu}\right)}$ at their effective region holds to the accuracy better than $0.1 \%$.) At large $\tan ^{2} \theta_{13} R \simeq 0$ as it should be for $\nu_{\mu} \rightarrow \nu_{\tau}$ dominant region.

In Fig. 1(a) One notices that mountains are peaking out at $\theta_{23} \simeq \frac{\pi}{4}$ and $\theta_{13} \gtrsim \frac{\pi}{4}$. This feature can be understandable most easily at large $\tan ^{2} \theta_{13}$ and $\theta_{12}=\theta_{13}=\pi / 4$, where neutrinos can be made effectively unmixed after redefining the mass eigenstates. Therefore, both the numerator and the denominator of $\mathrm{R}$ are small and can have large ratios. If we vary $\theta_{12}$ away from $\pi / 4$ the peak position moves to different values of $\tan ^{2} \theta_{23}$ but the peak does not disappear. The feature may be regarded as a drawback of our definition of mixing angles in (11) in discussing the $\nu_{\mu} \rightarrow \nu_{\tau}$ dominant region.

Since we are interested in the region with sizable $\nu_{\mu}$ deficit we want to avoid to have a large $R$ value due to the artifact of the small denominator. Therefore, we impose the constraint $1-P\left(\nu_{\mu} \rightarrow \nu_{\mu}\right) \geq 0.1$ in our subsequent analysis. Namely, we set $R=0$ whenever the constraint is not met. We note that $10 \%$ deficit in probability amount to, very roughly speaking, more than $3 \sigma$ away from the Kamiokande results [5] which indicates about $40 \%$ deficit in the double ratio $\left(\frac{\nu_{\mu}}{\nu_{e}}\right)_{\text {observed }} /\left(\frac{\nu_{\mu}}{\nu_{e}}\right)_{\text {expected }}$.

The effect of the "cut" is displayed in Fig. 1(b); the mountains disappear. The cut also affects small- $\tan ^{2} \theta_{13}$ and large- $\tan ^{2} \theta_{23}$ region, the region of almost pure $\nu_{e} \rightarrow \nu_{\tau}$ oscillation. In fact, the ratio $R$ defined in (2) is not quite appropriate in discussing the $\nu_{e} \rightarrow \nu_{\tau}$ dominant region where $R$ is given by a ratio of two small numbers and hence its value is unstable. To explore this region of mixing angles we would need to repeat the similar consideration by using $\nu_{e}$ beam. In this paper we will confine ourselves to the $\nu_{\mu}$ experiment which is motivated by the atmospheric neutrino anomaly, leaving the discussion of $\nu_{e}$ experiment elsewhere.

The ratio $R$ defined in (2) has a number of good features. It is independent of the absolute normalization of the neutrino flux which is difficult to determine in a good accuracy 
experimentally. It is very stable with respect to changes of the parameters such as $\Delta m^{2}$ and the CP-violating angle $\delta$. We have checked that the Fig. 1 barely changes even if we take larger $\Delta m^{2}$, e.g., $\Delta m^{2}=5 \mathrm{eV}^{2}$ instead of $\Delta m^{2}=10^{-2} \mathrm{eV}^{2}$. The change is largest in regions with slopes, and smallest at the top and the bottom of the hill, the regions where are of interest to us. (See below.) But even the change in the former region is less than $0.1 \%$, indicating the remarkable stability of the ratio $R$. We will later come back to the issue of stability against the change toward smaller value of $\Delta m^{2}$ and inclusion of the CPviolating angle. Until it will be done the value of $\Delta \mathrm{m}^{2}$ is fixed as $10^{-2} \mathrm{eV}^{2}$ throughout the computation in this work.

In Fig. 2 we aim to present a global view of the equi- $R$ contours computed by varying the remaining angle $\theta_{12}$ from $10^{\circ}$ to $80^{\circ}$ in $1^{\circ}$ step at each point on the $\tan ^{2} \theta_{13}-\tan ^{2} \theta_{23}$ plane. In the upper half plane $\left(\tan ^{2} \theta_{13}>1\right)$ the contours of maximum values of $R$ obtained by varying $\theta_{12}$ are plotted for a given values of $\tan ^{2} \theta_{13}$ and $\tan ^{2} \theta_{23}$. In the lower half plane $\left(\tan ^{2} \theta_{13}<1\right)$ the contours of minimum values of $R$ obtained by the same procedure are plotted. These prescriptions are to obtain the most conservative bound on $R$ in the respective regions of $\tan ^{2} \theta_{13}$. The contours are drawn in 0.1 units covering the region $R=0.1-0.9$. The correspondence between various symbols for lines and the contours are again represented in the figure.

One notices that there are two regions which receive an artificial erosion by the cut $1-P\left(\nu_{\mu} \rightarrow \nu_{\mu}\right) \geq 0.1$ imposed; one at large $\tan ^{2} \theta_{13}$ and relatively small $\tan ^{2} \theta_{23}$, the other at small $\tan ^{2} \theta_{13}$ and large $\tan ^{2} \theta_{23}$. The restriction to $10^{\circ}$ to $80^{\circ}$ for the angle $\theta_{12}$ is due to the fact that unless the restriction is imposed extra regions which do not meet the cut criterion are created, the situation outside of our physical interest.

In Fig. 2 (and in Fig. 3) drawn by the thick lines are the constraints at the $90 \%$ CL imposed by the reactor and the accelerator experiments obtained by Fogli, Lisi and Scioscia [10]. They are for $\Delta M^{2}=5 \mathrm{eV}^{2}$. A comment is in order on the choice of this value. We want to derive the most conservative bound on the ratio $R$. Since the above constraint is milder for smaller $\Delta M^{2}$ we have to estimate the lowest possible value of the mass of the 
dark matter neutrinos. We use $m_{\nu}=23.4 \Omega_{\nu} h_{50}^{2} / N_{\nu}$ [1], where $\Omega_{\nu}$ is the fraction of neutrinos of the critical density, $N_{\nu}$ the number of species of dark matter mass neutrinos and $h_{50}$ denotes the Hubble parameter measured in units of $50 \mathrm{~km} / \mathrm{s} \cdot \mathrm{Mpc}$. We take a typical value $\Omega_{\nu}=0.2$, the "lowest" value $h_{50}=1$, and $N_{\nu}=2$ to minimize $m_{\nu}$ within our ansatz of hierarchical mass pattern. We obtain $m_{\nu}=2.34 \mathrm{eV}$, hence our choice of $\Delta M^{2}=5 \mathrm{eV}^{2}$.

For our purpose of making quantitative statements on the ratio $R$ we need to know the detailed structure of the contours at $R \lesssim 0.1$ and at $R \gtrsim 0.9$. It is presented in Fig. 3 where the equi- $R$ contours are drawn in 0.01 steps. The result presented in Fig. 3 quantifies our rough statement that the ratio $R$ must lie either close to zero or to unity in the presence of dark matter neutrinos; it must exist in restricted regions either $R<0.02$ or $R>0.87$, if the neutrinos come with only three flavors and the dark matter neutrinos have hierarchical mass pattern.

We examine here the stability of $R$ against inclusion of the CP-violating angle and variation of $\Delta m^{2}$. We have verified, by computing the cases $\delta=\pi / 4, \pi / 2$ and $\pi$, that no appreciable change exists in equi- $R$ contours in the allowed regions of the mixing parameter plane. We have also checked that there is no detectable difference in the contours of $R$ in the allowed mixing parameter regions up to $\Delta m^{2}=5 \times 10^{-3} \mathrm{eV}^{2}$. They cover interesting regions of $\Delta m^{2}$ as a solution of the atmospheric neutrino problem.

A remark on the possibility of using other oscillation channels than $\nu_{\mu} \rightarrow \nu_{e}$ is in order. If the appearance measurement $\nu_{\mu} \rightarrow \nu_{\tau}$ is feasible, for instance in MINOS [14] and ICARUS 15] experiments, one can do the same job by defining the similar ratio $R_{\tau}$ as $R_{\tau}=$ (No. of observed $\tau$ events)/ (No. of expected muon events - No. of observed muon events). One then expect $R_{\tau} \sim 0$ at $\nu_{\mu} \rightarrow \nu_{e}$ dominant region and $R_{\tau} \sim 1$ at $\nu_{\mu} \rightarrow \nu_{\tau}$ dominant region.

Finally we make a comment on the limitation of our framework, the three-flavor neutrino mixing without steriles. Since the two $\Delta m^{2}$ are used up as the dark matter and the atmospheric neutrino mass scales, it cannot accommodate the solar neutrino solution. However, there exist partial resolution of the problem. If we are in the $\nu_{\mu} \rightarrow \nu_{e}$ dominant region the scenario does imply the deficit in the solar neutrino flux in the manner of Acker and Pakvasa 
[16]. Namely, the various neutrino flux is reduced by factor of $\sim 2$ by the averaging effect due to the large $\Delta m^{2}$. On the other hand, if we are in the $\nu_{\mu} \rightarrow \nu_{\tau}$ region we would need to introduce a sterile neutrino which weakly mixes with $\nu_{e}$ and utilize the small-angle MSW mechanism [17.

It is difficult to make definitive statements on how our result would change when we include a sterile neutrino in a generic way. We have to look for the quantities which can quantify relative importance of $\nu_{\mu} \rightarrow \nu_{e}$ and $\nu_{\mu} \rightarrow \nu_{\tau}$ oscillations analogous to our $R$. But, the one quantity would not be enough. So the discussion on the utility of the long-baseline neutrino experiments as a probe of the dark matter neutrino is entirely open even in the minimal three-flavor + one-sterile case. Fortunately, some models indicate the feature that the three-flavor neutrino sector is perturbed only weakly by the inclusion of the sterile neutrinos [18]. If this is the case our consideration in this paper could still apply at least approximately to such models with extra sterile species.

We have demonstrated that the long-baseline neutrino oscillation experiments can probe into the dark matter neutrinos. They can put scheme-dependent (such as three-flavor mixing without steriles) constraint and are capable of rejecting broad parameter regions associated with the dark matter hypothesis. To quantify such test of the hypothesis we have introduced the ratio $R$ as a measure for relative importance of $\nu_{\mu} \rightarrow \nu_{e}$ and $\nu_{\mu} \rightarrow \nu_{\tau}$ oscillations.

We hope that the consideration in this paper urge long-baseline experimentalists even more strongly to design the experiments which allow them accurate measurement not only in the appearance but also in the disappearance channels.

We thank K. Nishikawa for informative correspondences on the long-baseline experiment KEK-PS $\rightarrow$ Super-Kamiokande. One of us (H.M.) has been supported in part by Grant-in-Aid for Scientific Research of the Ministry of Education, Science and Culture under \#0560355, and is supported by Grant-in-Aid for Scientific Research on Priority Areas under \#08237214. 


\section{REFERENCES}

[1] J. A. Holtzman, Astrophys. J. Suppl. 71, 1 (1989); J. A. Holtzman and J. R. Primack, Astrophys. J. 405, 428 (1993); J. R. Primack, J. Holtzman, A. Klypin, and D. O. Caldwell, Phys. Rev. Lett. 74, 2160 (1995); K. S. Babu, R. K. Schaefer, and Q. Shafi, Phys. Rev. D53, 606 (1996); D. Pogosyan and A. Starobinsky, astro-ph/9502019.

[2] For a review, see e.g., M. White and D. Scott, Comments Astrophys. 18, 289 (1966).

[3] D. Saltzberg, hep-ex/9606004, Talk presented at Seventh International Workshop on Neutrino Telescopes, Venice, Italy, March 1996.

[4] M. Laveder, hep-ph/9601342.

[5] K. S. Hirata et al., Phys. Lett. B205, 416 (1988); B280, 146 (1992); Y. Fukuda et al., ibid B335, 237 (1994).

[6] R. Becker-Szendy et al., Phys. Rev. D46, 3720 (1992).

[7] W. W. M. Allison et al, hep-ex/9611007.

[8] H. Minakata, Phys. Rev. D52, 6630 (1995); Phys. Lett. B356, 61 (1995).

[9] S. M. Bilenky, A. Bottino, C. Giunti, and C. W. Kim, Phys. Lett. B356, 273 (1995).

[10] G. L. Fogli, E. Lisi, and G. Scioscia, Phys. Rev. D52, 5334 (1995).

[11] C. Jarlskog, Phys. Rev. Lett. 55, 1039 (1985).

[12] K. Nishikawa et al., Proposal for a Long Baseline Neutrino Oscillation Experiment using KEK-PS and Super-Kamiokande, February 1995.

[13] M. Nakahata et al., J. Phys. Soc. Japan, 55, 3786 (1986).

[14] The MINOS Collaboration, MINOS Experiment R\&D Plan: FY 1996-1998, June 1996.

[15] The ICARUS Collaboration, ICARUS II, A Second-Generation Proton Decay Experiment and Neutrino Observatory at the Gran Sasso Laboratory, September 1993. 
[16] A. Acker and S. Pakvasa, Hawaii preprint, hep-ph/9611423.

[17] S. P. Mikheyev and A. Smirnov, Sov. J. Nucl. Phys. 42, 913 (1985); L. Wolfenstein, Phys. Rev. D17, 2369 (1978).

[18] A. Yu. Smirnov, Talk given at International Conference on High Energy Physics, Warsaw, July 1996. 


\section{FIGURE CAPTIONS}

Fig.1. The bird's-eye view of the global features of the ratio $R$ and its projection onto the plane spanned by $\tan ^{2} \theta_{13}$ and $\tan ^{2} \theta_{23}$. The remaining parameters are taken as $\theta_{12}=\pi / 4, \delta=0$ and $\Delta m^{2}=10^{-2} \mathrm{eV}^{2}$. The correspondence between various symbols and the equi- $R$ contours are exhibited in the figure. The figures (a) and (b) are for cases without and with constraint $1-P\left(\nu_{\mu} \rightarrow \nu_{\mu}\right) \geq 0.1$. (See the text.)

Fig.2. Equi- $R$ contours are plotted on $\tan ^{2} \theta_{13}-\tan ^{2} \theta_{23}$ plane. In the upper (lower) half plane, $\tan ^{2} \theta_{13}>1\left(\tan ^{2} \theta_{13}<1\right)$, the contours of maximum (minimum) values of $R$ obtained by varying $\theta_{12}$ are plotted for a given values of $\tan ^{2} \theta_{13}$ and $\tan ^{2} \theta_{23} . \Delta m^{2}$ is taken as $10^{-2} \mathrm{eV}^{2}$. The correspondence between various symbols and the equi- $R$ contours are represented in the figure. The thick solid lines are the constraints from reactor and accelerator experiments obtained by Fogli et al. 10.

Fig.3. The same as in Fig. 2 but the figure aims to display the detailed feature of the equi- $R$ contours at around the boundaries of the relevant parameter regions allowed by the terrestrial experiments. The large- $s_{13}^{2}$ and arbitrary $s_{23}^{2}$, and the small- $s_{13}^{2}$ and small- $s_{23}^{2}$ regions are presented in Fig.3(a) and Fig.3(b), respectively. The thick solid lines are the constraints from reactor and accelerator experiments obtained by Fogli et al. 10. 


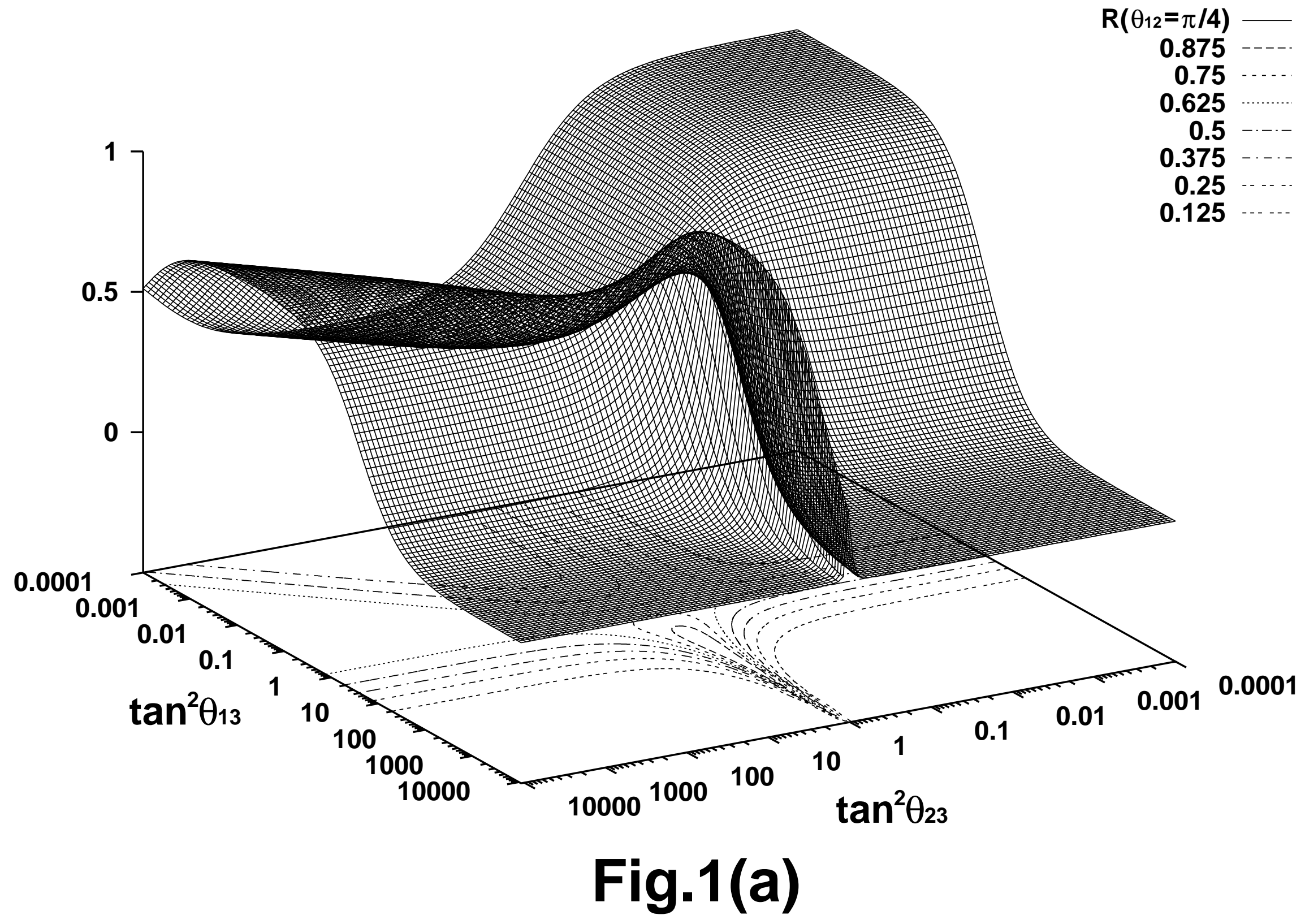




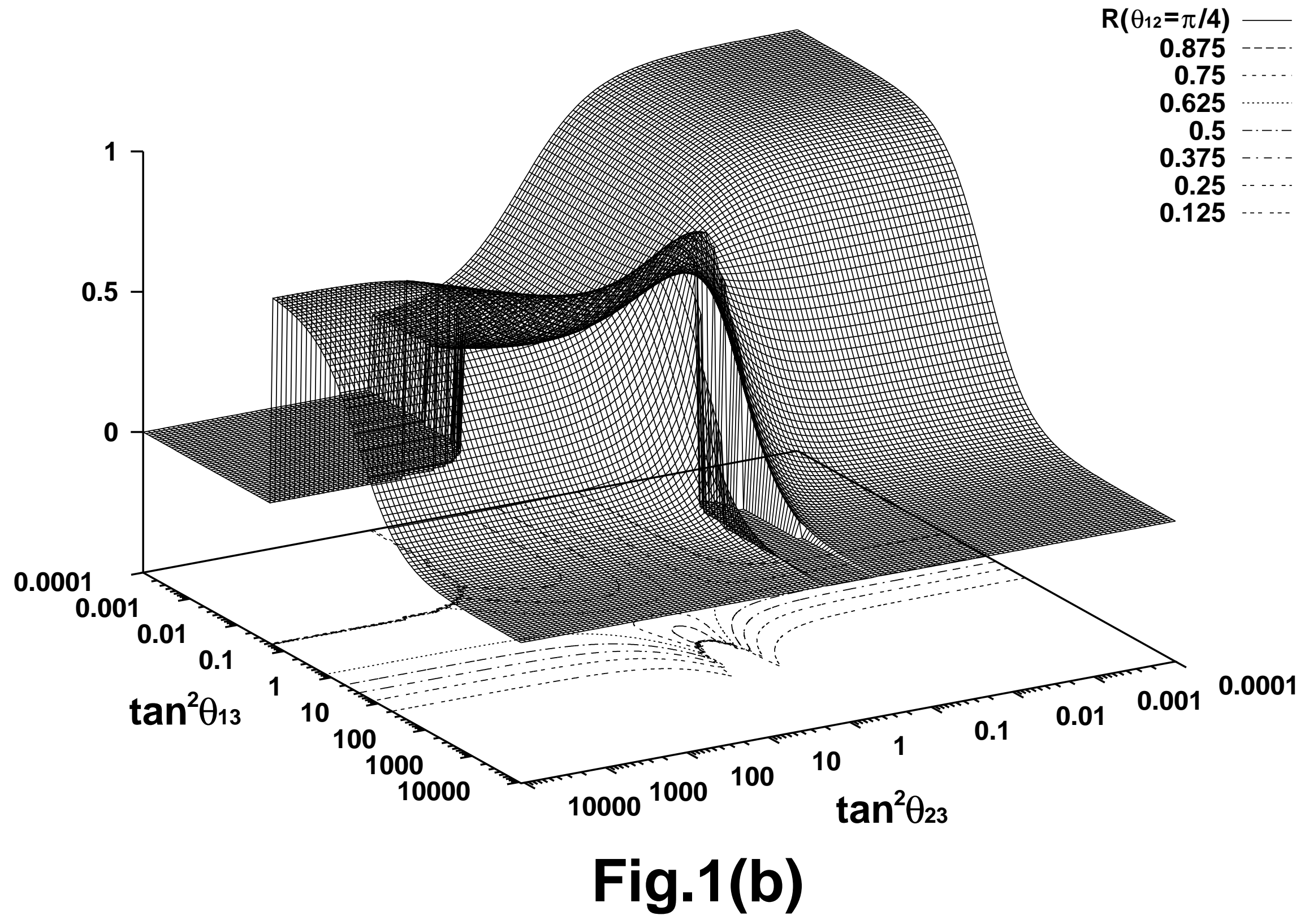




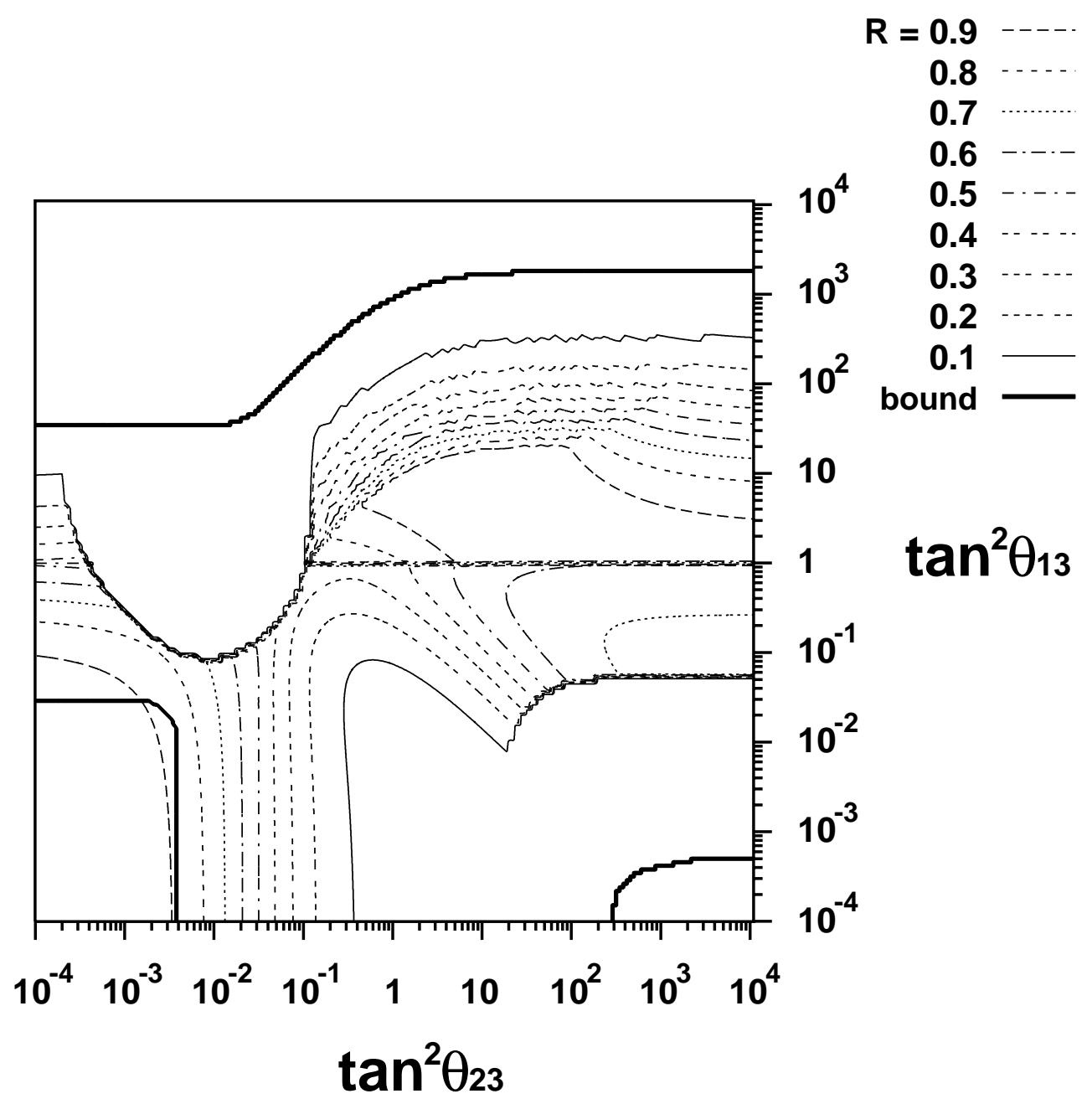

Fig.2 


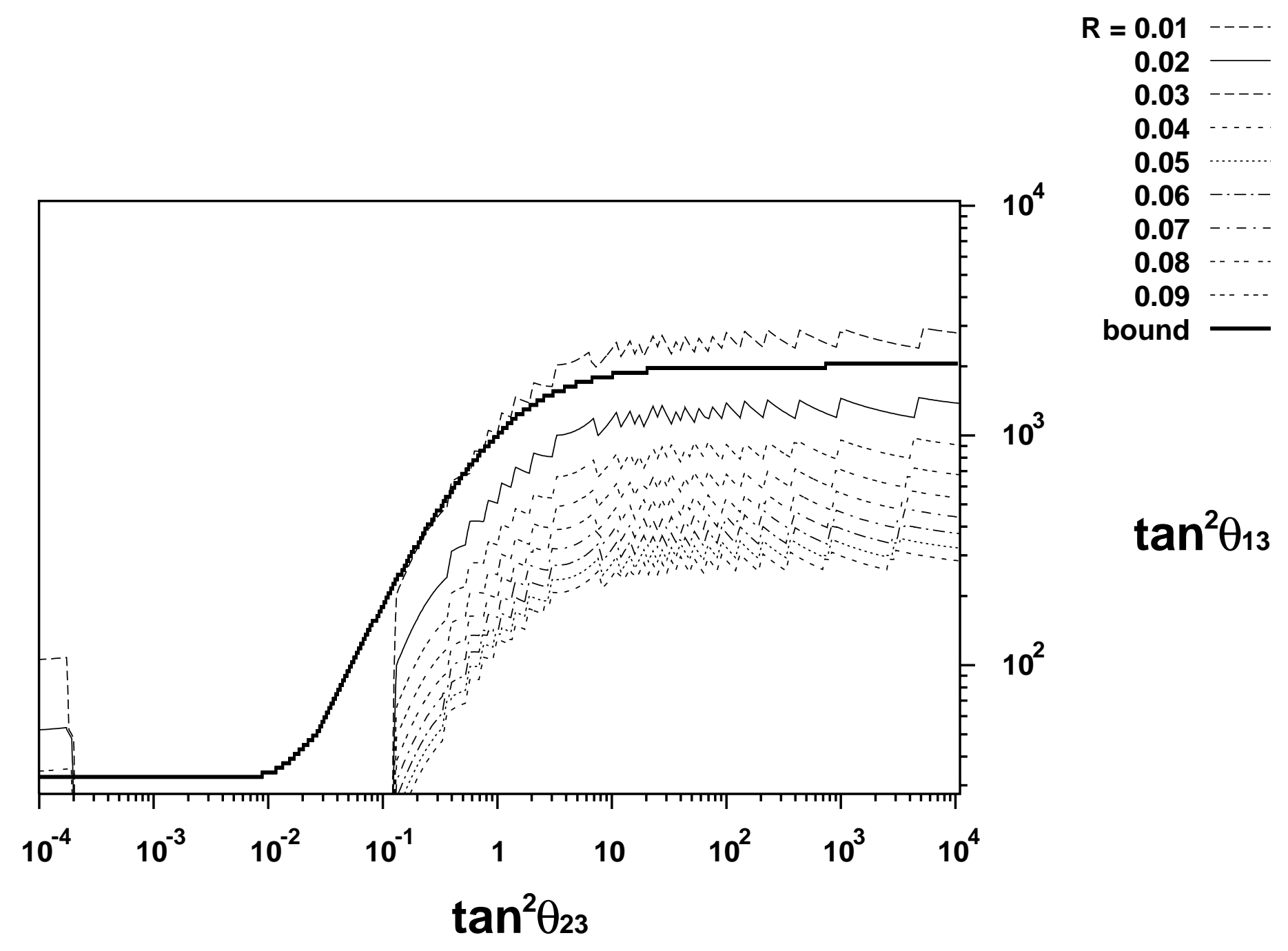

Fig.3(a) 
$R=0.90$

0.89

0.88

0.87

0.86

0.85

0.84

0.83

0.82 bound

0.01

$\tan ^{2} \theta_{13}$

0.001

0.0001

0.001

0.01

$\tan ^{2} \theta_{23}$

Fig.3(b) 